\title{
A new probiotic candidate bacterium from human milk: Limosilactobacillus vaginalis MA-10
}

\author{
M. Asan-Ozusaglam ${ }^{1 *}$ (1) and A. Gunyakti ${ }^{1,2}$ \\ ${ }^{1}$ Department of Biotechnology, Faculty of Science and Letters, Aksaray University, 68100, Aksaray, \\ Turkey \\ ${ }^{2}$ Institute of Biotechnology, Ankara University, 06110, Ankara, Turkey
}

\section{ORIGINAL RESEARCH PAPER}

Received: March 18, 2020 • Accepted: August 26, 2020

Published online: February 12, 2021

(C) 2020 The Author(s)

\begin{abstract}
The probiotic and technological potentials of lactic acid bacteria originating from human milk are becoming a remarkable research area. In the present study, Limosilactobacillus vaginalis MA-10 isolated from human milk was investigated in vitro for its probiotic and technological aspects. According to the results obtained in the study, MA-10 strain exhibited non-haemolytic activity and various degrees of sensitivity to most of the tested antibiotics. The strain showed good resistance to the gastrointestinal system and maintained its viability under these conditions. Its antimicrobial activity against human or clinical bacterial and fungal microorganisms and fish bacteria was determined in the range of $2.38-11.22 \mathrm{~mm}$. The MA-10 strain was able to assimilate cholesterol ranging from 31.42 to $82.30 \%$. The strain showed $9.34 \%$ ferrous-ion chelating and 32\% DPPH free radical scavenging activities. These initial results from the present study confirm that $L$. vaginalis MA-10 may be a new source with appropriated probiotic and technological traits for various industries, and further in vivo assays.Due to the limited number of studies on L. vaginalis strains originated from human breast milk in the literature, the data obtained in this study are thought to be important for revealing the basic probiotic properties of the strain.
\end{abstract}

\section{KEYWORDS}

human milk, antioxidant activity, cholesterol assimilation, gastrointestinal system

*Corresponding author. E-mail: meltemozusaglam@gmail.com 


\section{INTRODUCTION}

A healthy gastrointestinal tract microbiota is highly balanced in terms of the distribution of beneficial/harmful microorganisms, but the deterioration of this balance may lead to the onset of a pathological process called microbial dysbiosis and the development of symptoms related to it. The deterioration of commensal microbiota homeostasis causes a wide range of diseases. Most of the bacterial groups, such as Lactobacillus, Lactococcus, Bacillus, Bifidobacterium, which are naturally found in the gastrointestinal tract, are frequently used as food and health-promoting supplements to re-establish homeostasis (Kothari et al., 2019). Also, in recent years, human milk has gained considerable importance as safe and attractive source of probiotic lactic acid bacteria (LAB). The most commonly found and isolated bacteria species from human breast milk include Lactobacillus, Bifidobacterium, Streptococcus, and Enterococcus. Human milk bacteria are gaining much attention because of their ability to protect the host from pathogenic microorganisms, survive in the gastrointestinal tract, adhere to epithelial cells of the mucosa, assimilate cholesterol, support immune defence, produce antimicrobial substances and mediators (Amenu, 2014).

The use of LAB in food and feed industrial applications enjoys a "generally recognised as safe" (GRAS) status. Literature have focused on the Lactobacillus genus among LAB, and their probiotic effects were reported to be genus-specific, species-specific, or even strain-specific. In the literature, the research is generally focused on the probiotic potential of Lactobacillus gasseri, Lactobacillus fermentum, and Lactobacillus plantarum strains originated from human milk (Gunyakti and Asan-Ozusaglam, 2018, 2019). However, few studies have reported the probiotic potential and technological properties of Limosilactobacillus vaginalis isolated from human stomach, feces, vagina, and chicken. Therefore, the aim of the current study was to investigate L. vaginalis originated from human milk as a possibly potential probiotic candidate.

\section{MATERIALS AND METHODS}

\subsection{Identification of Lactobacillus strains}

The Gram-positive and catalase-negative isolate with bacillus morphology was first identified using API $50 \mathrm{CHL}$ (BiomerieuxSA, France) according to the manufacturer's instructions. The defined strain was then sent to IONTEK (Turkey) for molecular identification by $16 \mathrm{~S}$ rRNA PCR analysis.

\subsection{Determination of safety}

Haemolytic activity was verified by inoculating Colombia agar media containing $5 \%$ sheep blood (OR-BAK, Turkey) with the isolate and incubating under anaerobic condition at $37^{\circ} \mathrm{C}$ for $24 \mathrm{~h}$. Haemolytic activity was determined by zone formation around the colonies. The greenish zone was considered $\alpha$-haemolysis, clear zone was classified as $\beta$-haemolysis, and no zone indicated $\gamma$-haemolysis.

The susceptibility profiles of L. vaginalis MA-10 were assessed by the disc diffusion method against eleven commercial antibiotics (Oxoid, Germany). The suspension adjusted to Mc Farland 0.5 densities was spread on MRS agar medium. Each antibiotic disc was located on the 
inoculated MRS agar medium in triplicate, and the Petri dishes were incubated at $37^{\circ} \mathrm{C}$ for $24 \mathrm{~h}$. The inhibition zone diameters were measured and assessed as stated by CLSI (Clinical and Laboratory Standards Institute) guideline (Sharma et al., 2016).

\subsection{Determination of probiotic potential and technological properties}

2.3.1. Cell viability under simulated gastrointestinal conditions. The resistance of L. vaginalis MA-10 to simulated gastric conditions including low $\mathrm{pH}$, bile, gastric, and small intestine juice tolerances were determined as described in our previous study (Gunyakti and Asan-Ozusaglam, 2019).

2.3.2. Determination of antimicrobial activity. Fifteen clinical and food-borne bacteria, two fungal strains and seven fish originated pathogenic bacteria were used to determine the antimicrobial activity of $L$. vaginalis MA-10 using well diffusion method. The indicator microorganisms adjusted to McFarland 0.5 standard were spread onto the specific agar media. MA-10 strain supernatant $(100 \mu \mathrm{L})$ was placed into each well $(7 \mathrm{~mm}$ diameter). The plates were incubated for $24 \mathrm{~h}$ and then the inhibition zones around the wells were measured using Vernier caliper. The inhibition zone diameter was obtained by subtracting the diameter of the well from the entire inhibition diameter. The experiment was conducted three times.

2.3.3. Cholesterol assimilation activity. L. vaginalis MA-10 culture was inoculated into the MRS broth containing $100 \mu \mathrm{g} \mathrm{mL}^{-1}$ of cholesterol (Sigma-Aldrich, USA) and different concentrations of bile ( 0.3 and $1 \%$ ) (Oxoid, UK) then incubated at $37{ }^{\circ} \mathrm{C}$ for $24 \mathrm{~h}$. The cholesterol assimilation of supernatant and pellet was separately evaluated using the $o$-phthalaldehyde method (Hosono et al., 1999). Cholesterol removal ability of MA-10 was calculated as described in our previous study (Gunyakti and Asan-Ozusaglam, 2019).

2.3.4. Antioxidant activity. The free radical scavenging activity and ferrous-ion chelating property of MA-10 strain were measured using 2,2-diphenyl-1-picrylhydrazyl (DPPH) as described in our previous study (Gunyakti and Asan-Ozusaglam, 2019).

\subsection{Statistical analysis}

Statistical analysis was performed with the Mann-Whitney U-test to identify significant differences in antibiotic susceptibility and antimicrobial activity assay results. The differences were considered significant at a $P$-value of $<0.05$. The statistical analyses were conducted using SPSS version 22 (SPSS Inc, Chicago, IL, USA).

\section{RESULTS AND DISCUSSION}

\subsection{Identification of Lactobacillus strain}

The isolated strain was identified by API 50 CHL System as Lactobacillus acidophilus (98.7\%). Genotypic identification according to the 16S rRNA sequences showed that MA-10 strain was L. vaginalis. However, molecular identification with $16 \mathrm{~S}$ rRNA of strains may be more consistent 
than microbial and biochemical techniques. Therefore, the strain was identified as L. vaginalis MA-10 according to the $16 \mathrm{~S}$ rRNA PCR analysis results.

\subsection{Determination of safety}

The strain had no-zones around the colonies on the blood agar. L. vaginalis MA-10 exhibited $\gamma$ haemolytic or non-haemolytic activity. Haemolytic activity is related to virulence factors that can be found in pathogenic microorganisms, so the microorganisms under investigation for probiotic properties are expected to be non-haemolytic strains (FAO/WHO, 2002). In this study, the absence of a lysis region for MA-10 strain indicates the safety of this strain as a probable probiotic candidate in food and other application areas.

L. vaginalis MA-10 showed susceptibility to chloramphenicol, amoxicillin, erythromycin, and ampicillin and resistance to amikacin, gentamicin, kanamycin, nalidixic acid, and ofloxacin (Table 1). The intermediate resistance was determined against cloxacillin and penicillin G. There are mean differences in the antibiotic susceptibility of MA-10 strain against the tested antibiotics. The statistical analysis, however, indicated that the inhibition zone averages of MA-10 strain were not statistically significant atthe significance level of $0.05(P>0.05)$. The safety concerns related to the use of antibiotic-resistant strains as probiotics are due to the possibility of transferring antibiotic-resistance genes to pathogens in the intestine (Curragh and Collins, 1992). However, in the literature, the antibiotic resistance of some LAB is thought to be natural or intrinsic, so chromosomally encoded and thereby non-transmissible. For example, Bifidobacterium strains resistant to various antibiotics in quinolone and aminoglycoside groups are used as starter cultures in food applications. Resistance to aminoglycoside antibiotics, such as amikacin, kanamycin, streptomycin, andgentamicin, is considered to be intrinsic in the Lactobacillus genus. The reason for the resistance is the lack of electron transport mediated by the cytochrome, as it mediates the drug uptake (Danielsen and Wind, 2003). Literature information supports our study data, suggesting that $L$. vaginalis strain resistance genes are structural and the risk of transfer to other microorganisms is low. However, in later studies, these results can be confirmed by molecular-based methods.

\subsection{Determination of probiotic potential and technological properties}

3.3.1. Cell viability under simulated gastrointestinal conditions. The high tolerance to acidity at $\mathrm{pH} 2$ and 3 was found for MA-10 with a high survival rate after $3 \mathrm{~h}$ (Table 2). The survival rate was found to be over $100 \%$ for both bile concentrations during the incubation period $(4 \mathrm{~h}$ ) (Table 2). During fasting and after the meal, the $\mathrm{pH}$ ranges from 1.5 to 4.5 , and the food digestion is completed in about $3 \mathrm{~h}$ (Lebeer et al., 2008). MA-10 strain showed high survival rates at $\mathrm{pH} 3$. Delgado et al. (2015) reported almost similar growth rate for two $L$. vaginalis strains (LV121 and LV51) from the human stomach. Herein, MA-10 strain exhibited cell viability as 7.26 and $7.65 \mathrm{CFU} \mathrm{mL}^{-1}$ at $\mathrm{pH} 2$ and 3, respectively, after $3 \mathrm{~h}$. MA-10 strain showed higher viability at $\mathrm{pH} 2$. The strains that pass the acidic barrier of the stomach are then exposed to bile fluid. Therefore, tolerance to bile is another criterion to be a good probiotic. The strain has maintained its viability under different $\mathrm{pH}$ and bile conditions. 
Table 1. Antibiotic susceptibility profile of L. vaginalis MA-10

\begin{tabular}{|c|c|c|c|c|c|c|c|c|c|c|c|}
\hline & \multicolumn{11}{|l|}{ Antibiotics } \\
\hline & $\mathrm{AK}$ & $\mathrm{AMC}$ & $\mathrm{AM}$ & $\mathrm{C}$ & OB & E & $\mathrm{CN}$ & K & NA & OFX & $\mathrm{P}$ \\
\hline $\begin{array}{l}\text { Inhibition zone } \\
\text { diameter }(\mathrm{mm})\end{array}$ & $7.38^{\mathrm{b}} \pm 0.26$ & $26.11 \pm 0.91$ & $26.58 \pm 1.04$ & $25.31 \pm 0.93$ & $19.86 \pm 0.86$ & $26.08 \pm 1.36$ & $7.66 \pm 0.61$ & - & - & - & $19.68 \pm 1.56$ \\
\hline CLSI $^{\mathrm{a}}$ & $\mathrm{R}$ & $S$ & $S$ & $S$ & IR & $S$ & $\mathrm{R}$ & $\mathrm{R}$ & $\mathrm{R}$ & $\mathrm{R}$ & IR \\
\hline
\end{tabular}

- Indicates no inhibition zone.

${ }^{a}$ The inhibition zones was evaluated as Susceptible $(S)>20$, Intermediate $(\mathrm{IR}) \cong 15-19$, Resistant $(\mathrm{R}) \leq 14$.

${ }^{\mathrm{b}}$ Diameter of the inhibition zone including disc diameter. AK: Amikacin $(10 \mu \mathrm{g})$; AMC: Amoxicillin $(30 \mu \mathrm{g})$; AM: Ampicillin $(10 \mu \mathrm{g})$;

C: Chloramphenicol $(10 \mu \mathrm{g})$; OB: Cloxacillin $(5 \mu \mathrm{g})$; E: Erythromycin $(15 \mu \mathrm{g})$; CN: Gentamicin $(10 \mu \mathrm{g})$; K: Kanamycin $(30 \mu \mathrm{g})$; NA: Nalidixic Acid $(5 \mu \mathrm{g})$; OFX: Ofloxacin; P: Penicillin G $(10 \mu \mathrm{g})$. 
Table 2. Acid and bile tolerance of L. vaginalis MA-10

\begin{tabular}{|c|c|c|c|c|c|c|c|c|c|c|c|c|c|}
\hline \multicolumn{8}{|c|}{ Acid tolerance $\left(\log _{10} \mathrm{CFU} \mathrm{mL} \mathrm{mL}^{-1}\right)$} & \multicolumn{6}{|c|}{ Bile tolerance $\left(\log _{10} \mathrm{CFU} \mathrm{mL} \mathrm{m}^{-1}\right)$} \\
\hline \multicolumn{4}{|c|}{ pH 2} & \multicolumn{4}{|c|}{$\mathrm{pH} 3$} & \multicolumn{3}{|c|}{$0.3 \%$ bile } & \multicolumn{3}{|c|}{$1 \%$ bile } \\
\hline $0 \mathrm{~h}$ & $1 \mathrm{~h}$ & $3 \mathrm{~h}$ & $\begin{array}{l}\text { Survival } \\
\text { rate (\%) }\end{array}$ & $0 \mathrm{~h}$ & $1 \mathrm{~h}$ & $3 \mathrm{~h}$ & $\begin{array}{l}\text { Survival } \\
\text { rate }(\%)\end{array}$ & $0 \mathrm{~h}$ & $4 \mathrm{~h}$ & $\begin{array}{l}\text { Survival } \\
\text { rate }(\%)\end{array}$ & $0 \mathrm{~h}$ & $4 \mathrm{~h}$ & $\begin{array}{l}\text { Survival } \\
\text { rate (\%) }\end{array}$ \\
\hline 7.74 & 7.49 & 7.26 & 93.79 & 8.60 & 8.23 & 7.65 & 88.95 & 7.37 & 8.38 & 113.70 & 7.97 & 8.38 & 105.14 \\
\hline
\end{tabular}

Table 3. Simulated gastric and pancreatic juice tolerance of L. vaginalis MA-10

\begin{tabular}{|c|c|c|c|c|c|c|c|c|}
\hline \multicolumn{6}{|c|}{ Gastric juice } & \multirow{2}{*}{\multicolumn{2}{|c|}{$\begin{array}{c}\text { Pancreatic juice } \\
\left(\log _{10} \mathrm{CFU}\right. \\
\left.\mathrm{mL}^{-1}\right)\end{array}$}} & \multirow{3}{*}{$\begin{array}{l}\text { Survival } \\
\text { rate (\%) }\end{array}$} \\
\hline \multicolumn{2}{|c|}{$\begin{array}{c}\mathrm{pH} 2.0 \\
\left(\log _{10} \mathrm{CFU}\right. \\
\left.\mathrm{mL}^{-1}\right)\end{array}$} & \multirow{2}{*}{$\begin{array}{l}\text { Survival } \\
\text { rate (\%) }\end{array}$} & \multicolumn{2}{|c|}{$\begin{array}{c}\mathrm{pH} 3.0 \\
\left(\log _{10} \mathrm{CFU}\right. \\
\left.\mathrm{mL}^{-1}\right)\end{array}$} & \multirow{2}{*}{$\begin{array}{l}\text { Survival } \\
\text { rate (\%) }\end{array}$} & & & \\
\hline $0 \mathrm{~h}$ & $3 \mathrm{~h}$ & & $0 \mathrm{~h}$ & $3 \mathrm{~h}$ & & $0 \mathrm{~h}$ & $3 \mathrm{~h}$ & \\
\hline 10.35 & 10.54 & 101.83 & 9.95 & 9.57 & 96.18 & 10.02 & 10.09 & 100.69 \\
\hline
\end{tabular}

L. vaginalis MA-10 also showed good survival ability under simulated gastric and pancreatic conditions (Table 3). In general, most LAB seem to have natural survival ability in pancreatin, but contrary to pancreatin tolerance, bile tolerance is specific to the strain (Masco et al., 2007). L. vaginalis MA-10 showed $100.69 \%$ survival rate after $3 \mathrm{~h}$ under simulated gastric juice conditions at $\mathrm{pH} 2$ and 3. The results indicate that MA-10 can survive under gastrointestinal conditions and can thus be a probable candidate as probiotic strain.

3.3.2. Antimicrobial activity. Antimicrobial activities of MA-10 tested against 25 indicator strains are presented in Table 4. The inhibition zones of the food-borne and clinical bacterial strains varied from 2.38 to $11.22 \mathrm{~mm}$. The highest two inhibitory activities were found against Staphylococcus epidermidis ATCC 11228 and Listeria monocytogenes ATCC 7644. The strain inhibited the growth of two Candida strains and inhibition zone diameters were determined as 2.70 and $2.87 \mathrm{~mm}$. The growth of all fish originated pathogenic bacteria was also inhibited by MA-10 strain with inhibition zones ranging from 3.53 to $7.35 \mathrm{~mm}$. The highest antibacterial activity was determined against Aeromonas hydrophila. The statistical analysis indicated that the differences between zone averages of MA-10 were not statistically significant at the significance level of $0.05(P>0.05)$.

Anukam and Reid (2007) reported the antibacterial activity of vaginal L. vaginalis against Escherichia coli O157:H7 and Staphylococcus aureus as over $15 \mathrm{~mm}$ of inhibition zone diameter. In another study conducted by Siroli et al. (2017), vaginal L. vaginalis BC15, BC16, and BC17 showed antibacterial activities against L. monocytogenes ATCC 13932 with a 1 to $3 \mathrm{~mm}$ inhibition zone diameter. Herein, MA-10 strain showed higher antibacterial activity than these vaginal $L$. vaginalis strains against $L$. monocytogenes ATCC 7644 with $9.79 \mathrm{~mm}$ inhibition zone diameter. Intestinal colonisation or infection of the intestine with pathogenic microorganisms may cause a risk of necrotising enterocolitis that can be decreased with the colonisation with lactobacilli and bifidobacteria of the intestine (Reid et al., 2003). The antimicrobial activities of 
Table 4. Antimicrobial activity of L. vaginalis MA-10

\begin{tabular}{|c|c|}
\hline Test microorganisms & $\begin{array}{c}\text { L. vaginalis MA-10 } \\
\text { Inhibition zone diameter }(\mathrm{mm})\end{array}$ \\
\hline \multicolumn{2}{|l|}{$\begin{array}{l}\text { Human food borne and clinical } \\
\text { Bacterial strains }\end{array}$} \\
\hline E. coli ATCC 11229 & $3.76 \pm 0.08^{\mathrm{a}}$ \\
\hline E. coli $\mathrm{O} 157: \mathrm{H} 7$ & - \\
\hline B. megaterium (Pasteur Ins. 5117) & $3.52 \pm 0.03$ \\
\hline S. enteritidis RSKK 171 & $3.21 \pm 0.16$ \\
\hline B. cereus RSKK 863 & $3.25 \pm 0.66$ \\
\hline B. subtilis RSKK 244 & - \\
\hline E. coli ATCC 35218 & - \\
\hline M. luteus NRRL B-4375 & - \\
\hline S. enteritidis ATCC 13076 & - \\
\hline S. sonnei MU:57 & $2.38 \pm 0.27$ \\
\hline S. aureus ATCC 25923 & - \\
\hline Y. enterocolitica NCTC 11175 & - \\
\hline E. faecalis ATCC 29212 & - \\
\hline L. monocytogenes ATCC 7644 & $9.79 \pm 1.13$ \\
\hline S. epidermidis ATCC 11228 & $11.22 \pm 0.76$ \\
\hline \multicolumn{2}{|l|}{ Fungal strains } \\
\hline C. albicans ATCC 10231 & $2.70 \pm 0$ \\
\hline C. glabrata RSKK 04019 & $2.87 \pm 0.24$ \\
\hline \multicolumn{2}{|l|}{ Fish originated bacterial strains } \\
\hline$V$. alginolyticus & $4.64 \pm 0.57$ \\
\hline$V$. anguillarium $\mathrm{M} 1$ & $3.73 \pm 0.35$ \\
\hline V. anguillarium A4 & $3.53 \pm 0.31$ \\
\hline A. hydrophila ATCC 19570 & $7.35 \pm 0.78$ \\
\hline L. garviae & $4.44 \pm 0.45$ \\
\hline Y. ruckeri & $4.88 \pm 0.73$ \\
\hline S. agalactiae Pas. Inst. 55118 & $4.71 \pm 0.76$ \\
\hline
\end{tabular}

- Indicates no antimicrobial activity.

${ }^{\mathrm{a}}$ Diameter of the inhibition zone including disc diameter.

probiotic strains are necessary to prevent infection or invasion of pathogenic microorganisms. Various LAB are known to synthesize various inhibitory agents such as bacteriocins, organic acids, but in this study, it was observed that the inhibitory activity was lost at neutral $\mathrm{pH}$. Therefore, the inhibitory activity in the current situation can be ascribed to organic acid-like metabolic products from L. vaginalis MA-10. These results indicated that MA-10 strain can be used as a feed supplement in aquaculture in the form of single or co-culture with various probiotic microorganisms. Besides, the strain may be a new alternative bio-source that exhibits inhibitory activity against human pathogens.

3.3.3. Cholesterol assimilation activity. The cholesterol-reducing activity of MA-10 was determined in vitro in the presence of 0.3 and $1 \%$ bile, the results obtained were 44.69 and $31.42 \%$ for pellets and 82.30 and $81.19 \%$ for supernatants, respectively. The higher anticholesterol activity at both bile concentrations was obtained for supernatants compared to 
pellets. The increased bile concentrations caused a small decrease in cholesterol assimilation activities of the pellet and supernatant. The possible cause of cholesterol assimilation activities of Lactobacillus strains is to decrease the solubility of cholesterol and therefore reduce cholesterol intake (Oh et al., 2018). The high serum cholesterol level can be a risk factor for cardiovascular disease; therefore, cholesterol-reducing ability has become an important functional feature for the selection of probiotic strains. There is no literature data on the cholesterol-lowering activity of $L$. vaginalis according to our knowledge. Nowadays, the development of cholesterol-lowering bio-therapeutics has become an important area. L. vaginalis MA-10 can assimilate with good cholesterol-lowering percentages and, therefore, can be a new bio-agent to reduce cholesterol level.

3.3.4. Antioxidant activity. The antioxidant activity was evaluated by DPPH free radical scavenging and ferrous-ion chelating activities. The strain showed $32 \% \mathrm{DPPH}$ free radical scavenging and $9.34 \%$ ferrous-ion chelating activities. Many LAB have special systems to prevent the organisms from the reactive oxygen species. The activities of various LAB to reduce reactive oxygen species have been induced by many in vitro experiments. However, there are a limited number of studies on L. vaginalis strains. Delgado et al. (2015) tested the total antioxidant activity of L. vaginalis LV51 and LV121 strains and found $21 \%$ and no activity. Several synthetic antioxidants widely used in food, cosmetic, and medicinal industries are carcinogenic and may cause liver damage (Saduakhasova et al., 2013). Antioxidant activity of the probiotic bacteria plays an important role in the protection of host health from free radicals. Therefore, $L$. vaginalis MA-10 can be an alternative to the synthetic antioxidants as a natural antioxidant source.

\section{CONCLUSIONS}

In the current study, probiotic potential and some technological properties of L. vaginalis MA10 isolated from human milk were examined in vitro. MA-10 strain exhibited appropriate probiotic effects including non-haemolytic activity and high tolerance to gastrointestinal conditions. The results of the antimicrobial, antioxidant, and anti-cholesterol activity assays also indicated that MA-10 strain can beneficially affect host health with these properties. Considering the results of the current study, L. vaginalis MA-10 strain may be a new bio-source for food, feed, and pharmaceutical industries after confirming its benefits and safe use in animal models.

\section{REFERENCES}

Amenu, D. (2014). Probiotic properties of lactic acid bacteria from human milk. Journal of Medical Microbiology and Diagnosis, S3: 005.

Anukam, K.C. and Reid, G. (2007). Lactobacillus plantarum and Lactobacillus fermentum with probiotic potentials isolated from the vagina of healthy Nigerian women. Research Journal of Microbiology, 2: 81-87.

Curragh, H.J. and Collins, M. (1992). High levels of spontaneous drug resistance in Lactobacillus. Journal of Applied Bacteriology, 73: 31-36. 
Danielsen, M. and Wind, A. (2003). Susceptibility of Lactobacillus spp. to antimicrobial agents. International Journal of Food Microbiology, 82: 1-11.

Delgado, S., Leite, A.M., Ruas-Madiedo, P., and Mayo, B. (2015). Probiotic and technological properties of Lactobacillus spp. strains from the human stomach in the search for potential candidates against gastric microbial dysbiosis. Frontiers in Microbiology, 5: 766.

FAO/WHO (2002). WHO Working Group on Drafting Guidelines for the Evaluation of Probiotics in Food. Guidelines for the evaluation of probiotics in food: Report of a Joint FAO/WHO Working Group on Drafting Guidelines for the Evaluation of Probiotics in Food, London, Ontario, Canada, April 30 and May 1. https://www.who.int/foodsafety/fs_management/en/probiotic_guidelines.pdf (Accessed: 26 August 2020).

Gunyakti, A. and Asan-Ozusaglam, M. (2018). Investigation of the potential use of Lactobacillus gasseri originated from human milk as food additive. LWT - Food Science and Technology, 93: 613-619.

Gunyakti, A. and Asan-Ozusaglam, M. (2019). Lactobacillus gasseri from human milk with probiotic potential and some technological properties. LWT - Food Science and Technology, 109: 261-269.

Hosono, A. (1999). Bile tolerance, taurocholate deconjugation, and binding of cholesterol by Lactobacillus gasseri strains. Journal of Dairy Science, 82: 243-248.

Kothari, D., Patel, S., and Kim, S.K. (2019). Probiotic supplements might not be universally-effective and safe: a review. Biomedicine \& Pharmacotherapy, 111: 537-547.

Lebeer, S., Vanderleyden, J., and De Keersmaecker, S.C. (2008). Genes and molecules of lactobacilli supporting probiotic action. Microbiology and Molecular Biology Reviews, 72: 728-764.

Masco, L., Crockaert, C., Van Hoorde, K., Swings, J., and Huys, G. (2007). In vitro assessment of the gastrointestinal transit tolerance of taxonomic reference strains from human origin and probiotic product isolates of Bifidobacterium. Journal of Dairy Science, 90: 3572-3578.

Oh, N.S., Joung, J.Y., Lee, J.Y., and Kim, Y. (2018). Probiotic and anti-inflammatory potential of Lactobacillus rhamnosus $4 \mathrm{~B} 15$ and Lactobacillus gasseri $4 \mathrm{M} 13$ isolated from infant feces. Plos One, 13: e0192021.

Reid, G., Jass, J., Sebulsky, M.T., and Mccormick, J.K. (2003). Potential uses of probiotics in clinical practice. Clinical Microbiology Reviews, 16: 658-672.

Saduakhasova, S., Kushugulova, A., Kozhakhmetov, S., Shakhabayeva, G., Tynybayeva, I., Nurgozhin, T., Marotta, F., and Zhumadilov, Z. (2013). Antioxidant activity of the probiotic consortium in vitro. Central Asian Journal of Global Health, 24: 115.

Sharma, P., Tomar, S.K., Sangwan, V., Goswami, P., and Singh, R. (2016). Antibiotic resistance of Lactobacillus sp. isolated from commercial probiotic preparations. Journal of Food Safety, 36: 38-51.

Siroli, L., Patrignani, F., Serrazanetti, D.I., Parolin, C., Palomino, N., Vitali, B.., and Lanciotti, R. (2017). Determination of antibacterial and technological properties of vaginal lactobacilli for their potential application in dairy products. Frontiers in Microbiology, 8: 166.

Open Access. This is an open-access article distributed under the terms of the Creative Commons Attribution-NonCommercial 4.0 International License (https://creativecommons.org/licenses/by-nc/4.0/), which permits unrestricted use, distribution, and reproduction in any medium for non-commercial purposes, provided the original author and source are credited, a link to the CC License is provided, and changes - if any - are indicated. 